\title{
A Review of Contemporary Teleaudiology: Literature Review, Technology, and Considerations for Practicing
}

\author{
Jinsook Kim ${ }^{1,2}$, Seungik Jeon ${ }^{1}$, Dokyun $\mathrm{Kim}^{1}$, and Yerim Shin ${ }^{1}$ \\ ${ }^{1}$ Department of Speech Pathology and Audiology, Graduate School, Hallym University, Chuncheon, Korea \\ ${ }^{2}$ Division of Speech Pathology and Audiology, College of Natural Sciences, Research Institute of Audiology and Speech Pathology, \\ Hallym University, Chuncheon, Korea
}

Received September 15, 2020

Revised October 16,2020

Accepted October 21, 2020

\author{
Address for correspondence \\ Jinsook Kim, FAAA, PhD \\ Division of Speech Pathology and \\ Audiology, College of Natural \\ Sciences, Hallym University, \\ 1 Hallymdaehak-gil, \\ Chuncheon 24252, Korea \\ Tel +82-33-248-2213 \\ Fax +82-33-256-3420 \\ E-mail_jskim@hallym.ac.kr
}

The scope of teleaudiology has been noted with telehealth due to Coronavirus disease (COVID-19) recently. As the notion has been around us for more than 20 years ever since 1999, it is necessary to perceive the knowledge accurately and prepare for the successful implementation of it. Therefore, the literature review including screening and diagnostic audiometry, cochlear implants and hearing aids, and aural rehabilitation, telecommunications technology regarding several fields of teleaudiology, and considerations for practicing were identified. Although overall internet-based audiological services showed benefits in terms of outcome and accessibility, uncertainties of cost-effectiveness, the optimal level of support, and a need for further studies of many aspects for teleaudiology has arisen. In the view of technology, the store-and-forward (asynchronous/hybrid) and a real-time (synchronous) methods were introduced with one applied and nine registered patents recorded from 2004 to 2020 for the invention of teleaudiology in the United States. Also, 10 checklists were suggested for planning teleaudiology practice from prior experience in hosting the teleaudiology program. Conclusively, it is hoped that this review sheds light on recognizing and improving the existing teleaudiology services and helps overcome the challenges faced in the era of pandemic and untact world to come.

J Audiol Otol 2021;25(1):1-7 Telecommunications technology.

\section{Introduction}

In the era of the fourth industrial revolution, advanced technology such as information and communication technology and ubiquitous connectivity would narrow down the gap between patients and health care providers. Inadvertently, the word 'telehealth' which means healthcare at a distance has been around us. The need for telehealth service has received attention due to Coronavirus disease, also known as COVID-19, caused by a newly discovered severe acute respiratory syndrome coronavirus 2 (SARS-CoV-2) since it is mainly transmitted through droplets generated when an infected person coughs, sneezes, or exhales [1].

This is an Open Access article distributed under the terms of the Creative Commons Attribution Non-Commercial License (https://creativecommons.org/licenses/by-nc/4.0/) which permits unrestricted non-commercial use, distribution, and reproduction in any medium, provided the original work is properly cited.
For promoting access to medical care between the consumer and the healthcare professional via telecommunications technologies (also referred to as telemedicine, telehealth, and eHealth) in the USA, a non-profit organization American Telemedicine Association was established in 1993 [2]. In Korea, the policy for telemedicine has become unacceptable currently, but the necessity of creation for environment and the successful implementation of it was proposed for future preparation [3]. Although telemedicine is frequently used as a synonym of telehealth, it can be defined as using telecommunications technologies to support the delivery of all kinds of medical, diagnostic, and treatment-related services by doctors. Whereas telehealth stands for a wider variety of remote healthcare services beyond the doctor-patient relationship including services provided by nurses, pharmacists, social workers, and other healthcare providers for patients and their caregivers. Out of those services, the full scope of hearing care by 
the healthcare professionals is called teleaudiology [4].

The term teleaudiology was first used by Dr. Gregg Givens in 1999 in reference to a system being developed at East Carolina University in North Carolina, USA. And the first internet audiological test was accomplished in 2000 by Givens, Balch, and Keller [5]. The first transatlantic teleaudiology evaluation was performed in 2009 by Dr. James Hall who evaluated a patient in South Africa from Dallas, USA at the American Academy of Audiology conference. Since then, numerous new works have appeared in areas of diagnosis, pediatric audiology, rehabilitation, cochlear implants (CIs), neonatal hearing screening, and hearing aids (HAs) [6]. Teleaudiology, a way of providing valid service over considerable distance, allowed patients to have easy access to a wider array of hearing health care services. However, such a level of service and knowledge has not been always available to the whole world. Therefore, this study was aimed to identify the state of the art information of teleaudiology regarding literature review, telecommunications technology, and considerations for practicing in broad spectrum services.

\section{Literature Review}

The field of teleaudiology grew after the publication of the systematic review for 26 articles from 1997 to 2009 with audiological screening, audiological diagnosis, audiological intervention, and patient/clinician perceptions showing the scope of utilization. The screening procedures for infants, children, and adults confirmed the feasibility and reliability of teleaudiology service. The diagnostic applications including audiometry, video-otoscopy, otoacoustic emissions (OAEs), and auditory brainstem response (ABR) demonstrated the clinical equivalency to the conventional face-to-face version. For intervention studies, HA verification, counseling, and internet-based treatment for tinnitus indicated the reliability and effectiveness when compared to the conventional method. The authors concluded that teleaudiology held significant promise but required considerable empirical research to identify future implementation spans [4]. As teleaudiology had become an accessible and valid paradigm in general and numerous new works had appeared, a review of teleaudiology was performed again in 2016 [6]. Contemporary literature review of teleaudiology was performed on the basis of three subjects, screening and diagnostic audiometry, CIs and HAs, and aural rehabilitation briefly. Including recent researches, the articles were selected from the multiple electronic databases.

\section{Screening and diagnostic audiometry}

After the idea of screening service in very young children using telehealth medium for supporting rural universal newborn hearing screening (UNHS) program was first suggested in 2005 [7], preschool and school-aged children's screening services were explored in 2011 and 2016 [8,9]. For UNHS, objective hearing tests including distortion product OAEs (DPOAEs) and ABRs were performed using remote computing technology or a mobile van equipped with satellite technology in India [10], Guam [11], and USA [12] reducing loss to followup rate. For preschool children, otoscopy, pure-tone testing with play audiometry, tympanometry, and DPOAE were conducted using remote computing technology in 2011 [8]. For schoolaged children, remote hearing screening at a rural elementary school was first tried in 2008 and various innovative systems for effective school audiometric screening were utilized from 2014 to 2015 [9,13,14]. Also, new audiology patient pathways through online hearing screening and assessment were provided by a free online digit-in-noise test [15]. For CI candidates, routine audiometry, word recognition testing, Arizona Biological test, and consonant-nucleus-consonant test were examined to adapt teleaudiological technology and accepted to audiologists and patients [16].

An interesting development of a self-diagnostic system of a high-frequency audiometer for monitoring the ototoxicity was first described in 2012 and was updated for monitoring cisplatin management of veterans undergoing cancer treatment in 2015 [17]. The use of a self-screening paradigm was used by over 38,000 individuals with a minimal fee of US $\$ 5$ and found that $81 \%$ of screened individuals had hearing loss [18]. Although many researchers described the convenience of self pure-tone hearing test systems using home computers, the validation issues relating to the calibration became questionable [19]. However, smartphones demonstrated little variability of testing output levels and could be calibrated by model for reproducible intensity levels for tonal stimuli [20]. When a tablet-based automated hearing screening was performed to 244 school children, the highest diagnostic values were obtained and proved to be an improved hearing screening protocol [21].

\section{$\mathrm{Cl}$ and $\mathrm{HAs}$}

Since the first report of remote computing CI mapping in 2006 [22], a few studies have described the feasibility of CI mapping. For example, the typical CI mapping and tuning services were performed to 70 individuals with remote computing software and interactive video systems and reported that there was no difference between face-to-face and remote methods except lipreading in Germany [23]. Another project including 41 technology supporting staff and 94 CI users was investigated through the National Network of Teleaudiology 
at 20 communities in Poland and 1 community in Ukraine. This investigation was further developed to 316 patients with a more than $95 \%$ satisfaction rate and opened up new possibilities for CI patients [24,25]. For CI programming, two steps of teleconsultation were provided. The first step included pre-assessment, psychoacoustic, medical, audiological, and speech-language evaluations. The second step was the remote CI programming. Afterward, a questionnaire about rating the service, interaction with providers, and savings of time and cost was answered by each participant and found the high acceptance and efficiency of remote CI programming. The effectiveness of CI programming and aural rehabilitation was further validated by the retrospective study of 20 patients from 2015 to 2018 . Through this study, the similar and acceptable results between conventional and teleaudiologic methods were obtained with the high satisfaction rate [26]. In Korea, Korea Telecom (KT) and Yonsei University Health System established a 'KT telemedicine clinic' in Friongdong Hospital of Cambodia and carried out a remote CI mapping using a dedicated KT line [27].

For tuning a HA remotely, two telephone lines were utilized, one for tuning HA and the other for communicating between the client and clinician in 2003 [28]. When compared the control and experimental groups for validating this practice, outcomes were almost equal on nearly all measures for both groups except the slightly longer programming time for the experimental group indicating active involvement of the HA wearers. However, the teleconsultation services for HA fitting was found to be valid and effective [29,30]. A self-fitting HA system that could be implemented to teleaudiology was also examined and noticed the need for questionnaires and/or real-ear measurements for validation by several researchers [31].

\section{Aural rehabilitation}

After the pioneering work of internet-based self-help treatment of tinnitus in 2004, the hybrid solutions for providing needed aural rehabilitation and tinnitus therapy were further developed. That was an internet cognitive behavioral therapy program for tinnitus management and stress reduction including modules on understanding tinnitus, relaxation, positive imagery, and re-interpreting tinnitus with sound enrichment, sleep guidelines, and concentration [32,33]. The latter article provided an excellent blueprint for how tinnitus, aural rehabilitation, and balance therapy could be applied online. Inspired by these works, aural rehabilitation, tinnitus, psychological counseling, client adjustment to amplification, and auditory training have been investigated by many researchers with asynchronous paradigms. For example, a client received an email concerning aural rehabilitation and cognitive behavioral therapy then initiated interplay over the internet. Although overall internet-based audiological intervention showed benefits in terms of outcome and accessibility, uncertainties of cost-effectiveness, optimal level of support, and improving intervention compliance were observed as barriers [34] and a need for further studies of many aspects for teleaudiology services for rehabilitation had arisen. Also, competent and sustainable models were desired while the current electronic health services in audiology had focused on technical feasibility [35].

\section{Telecommunications Technology}

There are two types of telecommunications technology. One is the store-and-forward (asynchronous/hybrid) method, which performs audiological work to a patient and transfers the results via emailing or internet to a professional who will look at the results. The other is a real-time (synchronous) method, which performs audiological work to a patient in real-time as if a patient is sitting in front of a professional. For example, audiologists are used to testing patients remotely just like a patient in a sound booth while an audiologist sits outside the booth. This is virtually the same as testing a patient over the internet. The window is not a real glass window but a teleconference window. The only difference is that physical distance changed.

In the United States, one applied (2004/0073136A1), and nine registered patents were recorded from 2004 to 2020 for the invention of teleaudiology (Table 1). After patient application regarding multiple diagnostic hearing tests with ambient noise measurement, registered patents could be categorized into four categories as follows. Using a computer network to allow interaction between a test administration site and one or plurality of remote patient sites, the systems, five types of methods and associated devices performing diagnostic hearing tests were registered in 2005 (US 6,916,291 B2), 2009 (US 7,530,957 B2), 2010 (US 7,854,704 B2), 2012 (US 8,287,462 B2), and 2015 (US 8,777,869 B2). For monitoring and testing the hearing of inner ear function regarding ototoxicity with mathematical prediction algorithms, one patent was registered in 2016 (US 9,480,418 B2). For patient communications in remote hearing diagnostics, one invention got a patent regarding electrical circuitry for conditioning audio input and output signals as well as a means of switching paths for the audio data between equipment sources and personnel involved in a remote hearing testing session in 2018 (US 10,117,605 B2). This patient was held by the company, RemotEar, concerning remote diagnostics and hearing instrument programming in teleaudiology. The system provided the adapter that 
Table 1. The summary of the patents of the United States including title, number, inventor, and brief contents

\begin{tabular}{|c|c|c|c|}
\hline Title & Patent number & Inventor & Contents \\
\hline $\begin{array}{l}\text { Multiple diagnostic hearing } \\
\text { test with ambient noise } \\
\text { measurement }\end{array}$ & $\begin{array}{l}\text { US 2004/ } \\
0073136 \text { A1 }\end{array}$ & $\begin{array}{l}\text { A. Thronton } \\
\text { \& J. Harrison }\end{array}$ & $\begin{array}{l}\text { System and methods for real time hearing test with } \\
\text { ambient noise measurement }\end{array}$ \\
\hline $\begin{array}{l}\text { Diagnostic hearing assessments } \\
\text { distributed via the use of a } \\
\text { computer network }\end{array}$ & $\begin{array}{l}\text { US } 6,916,291 \text { B2 } \\
\text { US } 7,530,957 \text { B2 } \\
\text { US } 7,854,704 \text { B2 } \\
\text { US } 8,287,462 \text { B2 } \\
\text { US } 8,777,869 \text { B2 }\end{array}$ & $\begin{array}{l}\text { G. Givens, } \\
\text { \& D. Balch, et al. }\end{array}$ & $\begin{array}{l}\text { Using a computer network to allow interaction } \\
\text { between a test administration site and one or } \\
\text { plurality of remote patient sites, the systems, five } \\
\text { methods and associated devices performing } \\
\text { diagnostic hearing tests }\end{array}$ \\
\hline $\begin{array}{l}\text { System and methods for the } \\
\text { screening and monitoring of } \\
\text { inner ear function }\end{array}$ & US $9,480,418$ B2 & $\begin{array}{l}\text { S. Fausti, } \\
\text { \& R. Ellingson, } \\
\text { et al. }\end{array}$ & $\begin{array}{l}\text { Monitoring and testing the hearing of a user using } \\
\text { a portable unit }\end{array}$ \\
\hline $\begin{array}{l}\text { System, method and apparatus } \\
\text { for patient communications in } \\
\text { remote hearing diagnostics }\end{array}$ & US $10,117,605$ B2 & $\begin{array}{l}\text { D. Davis, } \\
\text { \& B. Lisiewski }\end{array}$ & $\begin{array}{l}\text { Patient communications system in remote hearing } \\
\text { diagnostics about input/output signal and audio } \\
\text { data of system }\end{array}$ \\
\hline $\begin{array}{l}\text { Real time, remote access to and } \\
\text { adjustment of patient hearing } \\
\text { aid }\end{array}$ & $\begin{array}{l}\text { US } 10,348,891 \text { B2 } \\
\text { US } 10,623,564 \text { B2 }\end{array}$ & D. Manchester & $\begin{array}{l}\text { Real-time, remote access to an adjustment of the } \\
\text { hearing aid of a patient while in a normal living } \\
\text { environment }\end{array}$ \\
\hline
\end{tabular}

could be easily inserted into a standard diagnostic equipment configuration. It enabled either permanent or temporary remote operation for testing and interactive counseling by a hearing care professional in an office setting. This technology platform enabled hearing care professionals to remotely administer diagnostic patient testing and HA adjustments recently, while a patient was located in a normal living environment. The real-time system that could access and adjust a HA remotely with cloud computing were registered in 2019 (US 10,348,891 B2) and 2020 (US 10,623,564 B2).

Several technologies were suggested through the courses of teleaudiology for educating the use of electronic information and telecommunications to support and promote longdistance clinical health, patient and professional health-related education, public health, and health administration in several universities. Those technologies included videoconferencing, internet, store-and-forward, streaming media, terrestrial and wireless communications. For example, the University of Texas educated the system of a clinic on a cart and the University of Alabama's program, 'Hear Here Alabama!' managed a rural hearing health study in the truck which was introduced exoterically at the website (https://www.audiologyonline.com/ articles/teleaudiology-101-19711). A mobile clinic in a large truck that took the clinic to the patient delivering synchronous services of teleaudiology was designed to house a mobile audiology clinic containing two audiology suites, one large booth, and another smaller booth. The truck functioned as a regular audiology clinic only on a much smaller scale. The larger booth had a typical audiology setup with video-otoscopy, audiometer, tympanometer, probe microphone, and hearing in- strument test box performing HA verification via real ear measurement system. In the smaller booth, they had a typical setup for a HA lab strictly used for testing adults (https:// www.ua.edu/ news/2015/08/uas-mobile-hearing-center-unveiled). Department of Veterans Affairs (VA) of the United States developed a unique cart for hearing care services for veterans called 'the clinic on the cart.' On this particular cart, an audiometer, a prefit system for fitting HA, a hearing instrument test box, and a tympanometer were attached. This system had two monitors. One was for communication between the patient and the audiologist visually and the other was for operating the software. Using the freefit collar, ambient noise assessor which monitored the background noise where the patient sat controlled the background noise by comparing room noise collected with room microphone to an American National Standards Institute (ANSI) standard. The frequency and transducer with the specific visual display indicating green as good and red as bad determined whether the room was quiet enough to meet ANSI standard at that moment. It also stored the status of that background noise for every stored threshold. The ambient noise assessor was originally developed for the VA but now is available for anyone who's looking to implement a teleaudiology program. And the whole figure was introduced exoterically at the website (https://www.audiologyonline.com/articles/teleaudiology-101-19711).

As an alternative to the traditional fitting and follow-up session, the teleaudiology tools by many HA companies are available and those are summarized by the types of service and specific functions (Table 2). For example, after initial fitting, a HA user can request assistance from his/her hearing care 


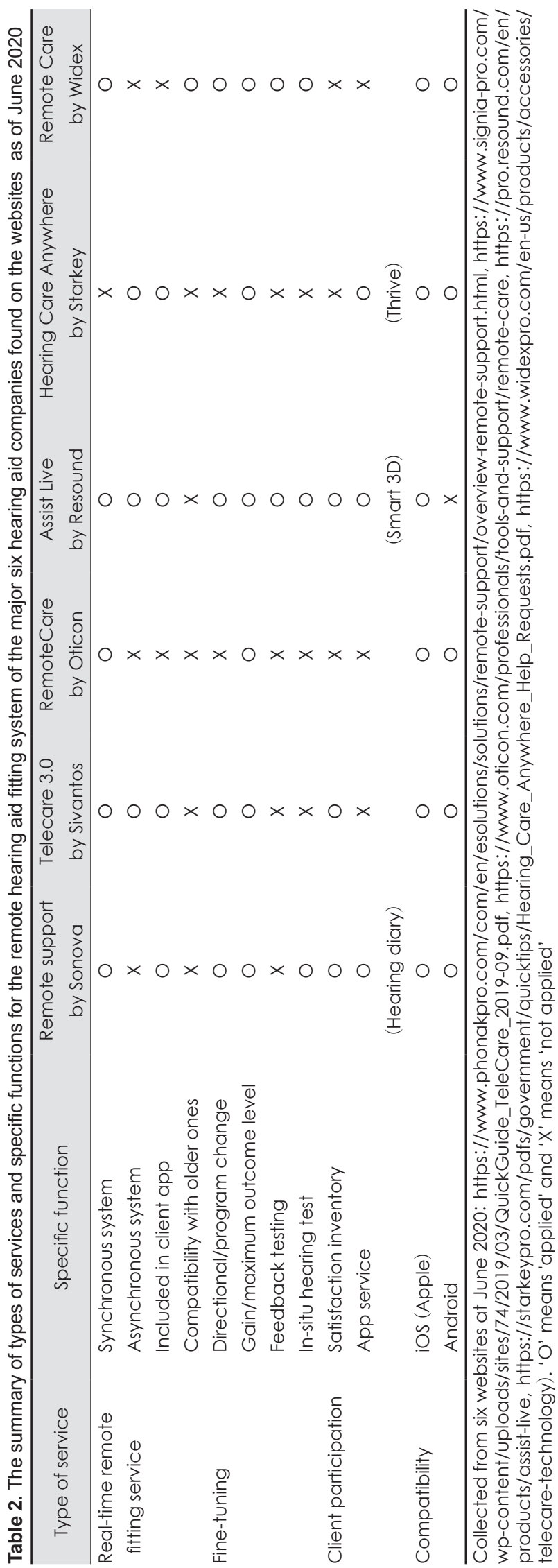

professional via the app on his/her smart device. The hearing care professional responds with a message and makes adjustments to the fitting and sends the adjustments back to the user as an update. Then the user can transfer this update to his/her HA via the wireless connection between the smart device and the HA. This application requires relatively little investment for the hearing care professional and user in terms of equipment and infrastructure. It offers a high degree of flexibility for a practice to implement and does not require a live interaction. The efficiency of this service was proved with a comparable quality of the service performed in person [36].

\section{Considerations for Practicing}

As changing perceptions of audiology and recognizing the importance of better hearing, early intervention and overall attention to the hearing-impaired population have increased. Consequently, greater demand has soared globally. While successful HA fittings depended on skilled hearing care professionals and their expertise, there was a shortage of hearing care providers in the United States to meet the future needs [37]. From prior experience in hosting the teleaudiology program in the United States, the checklist was culminated for getting started and implementing a structure to the existing facility. Ten items to consider for planning teleaudiology practice were suggested as follows: 1) Assess and confirm your readiness to start a teleaudiology program. This can be achieved by aligning itself with strength, weakness, opportunities, and threats analysis of the clinic or the organization to help justify the need for a teleaudiology component. This should be activated to identify the appropriate equipment and people to start the program and determine the types and levels of services to deliver. 2) Perform a market analysis and write a business plan. A business plan would help identify resources, allocation of funds, and approval from the related personnel. The plan should include detailed information on timelines, milestones, technical requirements, and potential challenges. 3) Build a team trained to forge ahead. All staff should be technically savvy and knowledgeable about the program. An application of the new technology and working together to create a seamless, comfortable, and reassuring clinical atmosphere for the patients is important. The provision of effective ongoing training and personnel development is necessary for increasing in scope and scale as the teleaudiology program expands. 4) Purchase the right equipment. Video equipment, communication systems with a high-speed internet connection, test equipment, and software programs/applications are critical 
components. Be mindful of the technology advance and budget. 5) Create an effective and professional environment that could emulate a traditional face-to-face encounter. The environment should be designed for effective videoconferencing considering room space, location, size, equipment, furniture placement, lighting, acoustics, patient privacy, and wall color for video background. The desirable atmosphere is user-friendly, well-equipped with reliable and appropriate technology, and comfortable for patients and staff. 6) Plan for the seamless integration of teleaudiology between sites. Alongside face-toface clinical activities, the new program should be designed to complement standard practices and working methods should not complicate or interrupt them. 7) Plan for information technology support at all participating locations. The expertise of equipment and network is essential and trained technical staff must be on hand to troubleshoot and make technical adjustments as necessary. 8) Establish protocols, policies, procedures, and short- and long-term performance goals. Written clinical and service protocols following standard recognizable protocols that lead to consistent clinical results should be available to all personnel involved in the teleaudiology program. 9) Develop an evaluation, monitoring, and quality improvement plan. It is recommended to implement an evaluation process to review the program and monitor its performance regularly. 10) Understand the legal and regulatory issues of teleaudiology. The legal and regulatory issues and requirements that must be understood and complied with can change periodically and must be implemented as required.

\section{Conclusion}

The benefits of teleaudiology are numerous. It has been identified at least four fundamental benefits: improved access, cost-effectiveness, improved quality, and patient demand. Given the provider shortages throughout the world in both rural and urban areas, teleaudiology has a unique capability to increase service to millions of patients who otherwise might not receive qualified hearing care. Teleaudiology has been shown to reduce the cost of hearing care and increase efficiency through better management of patients, shared clinic staff, reduced travel times/expenses, and high satisfaction rate. It can improve hearing care for many patients. Although we have not achieved the status of accommodating all the requirements, the new program could reduce travel time and related stress for patients, especially for those who have mobility issues. While there were personal security issues and legal regulation problems, the general attitude toward using teleaudiology was positive. In fact, one research reported a positive attitude among audiologists, when the acceptance towards the application of te- leaudiology was asked [38]. Therefore, we can conclude that the systematic review supports the use of teleaudiology for hearing care professionals and clients with hearing loss. However careful consideration of teleaudiology as a platform to deliver services is required due to the need for scaling up the management of hearing care professionals.

Finally, as the COVID-19 pandemic has resulted in physical distancing, teleaudiology as a derived area from telehealth is in the limelight [39]. The public opinion about telehealth can be identified by the recent media reports such as a 'Forbes' journal on the 5th of May 2020, "Telehealth. It's no longer just a nice-to-have, but instead a must-have for patients and healthcare professionals alike during these uncertain times the $\mathrm{CO}$ VID-19 pandemic has brought about. While we all wish that it hadn't taken a pandemic to propel telehealth forward, for better or for worse, it has. The spotlight is now on telehealth in a big way, and for good reason." (https://www.forbes.com/ sites/joeharpaz/2020/05/04/5-reasons-why-telehealth-hereto-stay-covid19/?sh=6b43193853fb) and a 'Foxbusiness' news report on 28th of May 2020, "Telehealth services, which are offered online, via phone and on mobile apps, allow health care professionals to conduct virtual visits with patients so they do not have to drive to an office. Many patients have been avoiding in-person visits during the coronavirus pandemic to reduce the spread of COVID-19." (https://www.foxbusiness. com/technology/how-does-telemedicine-work).

\section{Acknowledgments}

This work was supported by Hallym University Research Fund [HRF-2018O8-OO8] and the National Research Foundation of Korea grant funded from the Korea government Ministry of Science and ICT [2020R1F1A1069236].

\section{Conflicts of interest}

The authors have no financial conflicts of interest.

\section{Author Contributions}

Conceptualization: Jinsook Kim and Dokyun Kim. Data curation: Jinsook Kim and Seungik Jeon. Funding acquisition: Jinsook Kim. Investigation: All authors. Methodology: Jinsook Kim, Seungik Jeon, and Yerim Shin. Resources: Dokyun Kim. Supervision: Jinsook Kim. Validation: Jinsook Kim. Visualization: Seungik Jeon. Writing — original draft: Jinsook Kim, Seungik Jeon, and Dokyun Kim. Writing - review \& editing: Jinsook Kim, Seungik Jeon, and Yerim Shin. Approval of final manuscript: All authors.

\section{ORCID iDs}

Jinsook Kim

Seungik Jeon

Dokyun Kim

Yerim Shin https://orcid.org/0000-0003-3440-2393 https://orcid.org/0000-0003-2288-0750 https://orcid.org/0000-0003-3444-5246 https://orcid.org/0000-0002-2914-6711 


\section{REFERENCES}

1) Wikipedia, the free encyclopedia. Coronavirus disease 2019 [Internet]. Wikipedia; 2020 [cited 2020 Jul 30]. Available from: URL: https://en.wikipedia.org/wiki/Coronavirus_disease_2019.

2) Wikipedia, the free encyclopedia. Telehealth [Internet]. Wikipedia; 2020 [cited $2020 \mathrm{Jul}$ 30]. Available from: URL: https://en.wikipedia. org/wiki/Telehealth.

3) Kim JS, Oh SH, Choi JW, Kim SY. Current status of state telemedicine policy in the United States and policy implication for Korea. J Korean Med Assoc 2015;58:923-32.

4) Swanepoel de W, Hall JW 3rd. A systematic review of telehealth applications in audiology. Telemed J E Health 2010;16:181-200.

5) Wikipedia, the free encylopedia. Tele-audiology [Internet]. Wikipedia; 2018 [cited 2020 Aug 27]. Available from: URL: https://en.wikipedia.org/wiki/Tele-audiology.

6) Krumm M. A review of contemporary tele-audiology literature. J Hear Sci 2016;6:9-21.

7) Krumm M, Ribera J, Schmiedge J. Using a telehealth medium for objective hearing testing: implications for supporting rural universal newborn hearing screening programs. Semin Hear 2005;26:3-12.

8) Ciccia AH, Whitford B, Krumm M, McNeal K. Improving the access of young urban children to speech, language and hearing screening via telehealth. J Telemed Telecare 2011;17:240-4.

9) Skarzyński PH, Świerniak W, Piłka A, Skarżynska M, Włodarczyk $\mathrm{AW}$, Kholmatov D, et al. A hearing screening program for children in primary schools in Tajikistan: a telemedicine model. Med Sci Monit 2016;22:2424-30.

10) Ramkumar V, Hall JW, Nagarajan R, Shankarnarayan VC, Kumaravelu S. Tele-ABR using a satellite connection in a mobile van for newborn hearing testing. J Telemed Telecare 2013;19:233-7.

11) Hayes D, Boada K, Coe S. Early hearing detection and intervention by telepractice. Perspect Telepractice 2015;5:38-47.

12) Hayes D, Eclavea E, Dreith S, Habte B. From Colorado to Guam: Infant diagnostic audiological evaluations by telepractice. Volta Rev 2012;112:243-53.

13) Swanepoel de W, Myburgh HC, Howe DM, Mahomed F, Eikelboom $\mathrm{RH}$. Smartphone hearing screening with integrated quality control and data management. Int J Audiol 2014;53:841-9.

14) Botasso M, Sanches SG, Bento RF, Samelli AG. Teleaudiometry as a screening method in school children. Clinics (Sao Paulo) 2015;70: 283-8.

15) Ratanjee-Vanmali H, Swanepoel W, Laplante-Lévesque A. Characteristics, behaviors, and readiness of persons seeking hearing healthcare online. Int J Audiol 2019;58:107-15.

16) Fletcher KT, Dicken FW, Adkins MM, Cline TA, McNulty BN, Shinn JB, et al. Audiology telemedicine evaluations: potential expanded applications. Otolaryngol Head Neck Surg 2019;161:63-6.

17) Dille MF, McMillan GP, Helt WJ, Konrad-Martin D, Jacobs P. A store-and-forward tele-audiology solution to promote efficient screenings for ototoxicity during cisplatin cancer treatment. J Am Acad Audiol 2015;26:750-60.

18) Watson CS, Kidd GR, Preminger JE, Miller JD, Maki DP, Crowley A. Benefits of a telephone-administered national screening test. Audiology Online 2015;27:1-8.

19) Masalski M, Krecicki T. Self-test web-based pure-tone audiometry: validity evaluation and measurement error analysis. J Med Internet Res 2013;15:e71.

20) Masalski M, Kipiński L, Grysiński T, Kręcicki T. Hearing tests on mobile devices: evaluation of the reference sound level by means of biological calibration. J Med Internet Res 2016;18:e130.

21) Samelli AG, Rabelo CM, Sanches SGG, Martinho AC, Matas CG. Tablet-based tele-audiometry: automated hearing screening for schoolchildren. J Telemed Telecare 2020;26:140-9.

22) Franck K Pengelly M, Zerfoss S. Telemedicine offers remote cochlear implant programming. Volta Voices 2006;13:16-9.

23) Wesarg T, Wasowski A, Skarzynski H, Ramos A, Falcon Gonzalez JC, Kyriafinis G, et al. Remote fitting in Nucleus cochlear implant recipients. Acta Otolaryngol 2010;130:1379-88.

24) Wasowski A, Skarzynski, H, Lorens A, Obrycka A, Walkowiak A, Skarzynski $\mathrm{P}$, et al. The telefitting method used in the national network of teleaudiology: assessment of quality and cost effectiveness. J Hear Sci 2012;2:81-5.

25) Skarżyński PH, Świerniak W, Ludwikowski M, Bruski Ł. Telefitting between Kajetany and Odessa. Ukraine for cochlear implants. J Int Soc Telemed eHealth 2019;7:e17-1.

26) Luryi AL, Tower JI, Preston J, Burkland A, Trueheart CE, Hildrew DM. Cochlear implant mapping through telemedicine-A feasibility study. Otol Neurotol 2020;41:e330-3.

27) Kim Y. KT, established telemedicine at Cambodia [Internet]. Seoul: ZD Net Korea; 2018 [cited 2019 Jul 30]. Available from: URL: https:// zdnet.co.kr/view/?no=20181018142638.

28) Wesendahl T. Hearing aid fitting: application of telemedicine in audiology. Int Tinnitus J 2003;9:56-8.

29) Tao K. Teleaudiology hearing aid consultations for adults: towards innovating the service-delivery in clinical practice [dissertation]. Crawley (WA): The University of Western Australia; 2020.

30) Campos PD, Ferrari DV. Teleaudiology: evaluation of teleconsultation efficacy for hearing aid fitting. J Soc Bras Fonoaudiol 2012;24: $301-8$.

31) Keidser G, Convery E. Self-fitting hearing aids: status quo and future predictions. Trends Hear 2016;20:1-15.

32) Kaldo-Sandström V, Larsen HC, Andersson G. Internet-based cognitive-behavioral self-help treatment of tinnitus: Clinical effectiveness and predictors of outcome. Am J Audiol 2004;13:185-92.

33) Beukes EW, Manchaiah V, Allen PM, Baguley DM, Andersson G. Internet-based cognitive behavioral therapy for adults with tinnitus in the UK: Study protocol for a randomized controlled trial. BMJ Open 2015;5:e08241.

34) Beukes EW, Manchaiah V. Internet-based audiological interventions: an update for clinicians. Perspect ASHA Spec Interest Groups 2019; 4:542-52.

35) Ratanjee-Vanmali H, Swanepoel W, Laplante-Lévesque A. Patient uptake, experience, and satisfaction using web-based and face-toface hearing health services: process evaluation study. J Med Internet Res 2020;22:e15875.

36) Groth J, Dyrlund O, Wagener KC, Meis M, Krueger M. Industry research-fine-tuning outcomes are similar via teleaudiology and faceto-face. Canadian Audiologist 2019;6.

37) Ballachanda B. Critical steps in establishing a teleaudiology practice. Hearing Review 2017;24:14-7.

38) Ravi R., Gunjawate, DR, Yerraguntla K, Driscoll C. Knowledge and perceptions of teleaudiology among audiologists: a systematic review. J Audiol Otol 2018;22:120-7.

39) Henry JA, Thielman EJ, Kaelin C, Quinn CM, Goodworth MC. Telehealth-based progressive tinnitus management. The Hearing Journal 2020;73:32-3. 